\title{
THE VLA AND VLBA AT MILLIMETER WAVELENGTHS
}

\author{
EDWARD B. FOMALONT ${ }^{1}$ \\ National Radio Astronomy Observatory, 520 Edgemont Road, Charlottesville, \\ $V A$ 22903-2475, USA
}

\begin{abstract}
Although the Very Large Array (VLA) and the Very Large Baseline Array (VLBA) were originally intended as centimeter wavelength instruments, the exciting results from high resolution millimeter radio astronomy over the last ten years have generated interest in pushing these arrays into the millimeter region. This report will describe two aspects of recent development at NRAO: the new capability of the VLA at $7 \mathrm{~mm}$ wavelength which will be operational in 1994 , and the completion of the VLBA with its anticipated use at $7 \mathrm{~mm}$ and $3.6 \mathrm{~mm}$.
\end{abstract}

\section{THE VLA AT 7MM}

The VLA is located in New Mexico and consists of twenty-seven 25-m diameter antennas. They are arranged in a ' $Y$ '-configuration of four array sizes; $1 \mathrm{~km}, 3$ $\mathrm{km}, 10 \mathrm{~km}$ and $30 \mathrm{~km}$. The angular and frequency flexibility of the VLA make it an ideal instrument for many astronomical problems. The VLA was originally designed to operate in four frequency bands: $20 \mathrm{~cm}, 6 \mathrm{~cm}, 2 \mathrm{~cm}$ and $1.3 \mathrm{~cm}$. This frequency capability was extended to $90 \mathrm{~cm}, 400 \mathrm{~cm}$ ( 8 antennas), and $3.6 \mathrm{~cm}$ (for the JPL Viking encounter with Neptune).

The antennas of the VLA will soon have $7 \mathrm{~mm}$ receiver systems installed. The receiver construction is a collaboration between NRAO and the Instituto de Astronomia, Universidad Nacional Autonoma de Mexico, with financial support from Consejo Nacional de Ciencia y Tecnologia, Mexico. The receivers will contain HFET devices and are now being designed and proto-typed in Charlottesville, Va. The receiver temperature will be $40 \mathrm{~K}$ and the nominal system temperature should be $100 \mathrm{~K}$. The $7 \mathrm{~mm}$ system will interface with the normal IF system and correlator of the VLA, with dual frequency and dual circular polarization capabilities.

The first two antennas will be outfitted by August 1993 when array testing will begin. Six antennas should be completed by December 1993 when scientific observations in the A-configuration will commence. By May 1994, ten antennas will have the $7 \mathrm{~mm}$ system. The ten best antennas in terms of surface accuracy and pointing accuracy were chosen for the upgrade. An offset pointing scheme (adjust pointing on a strong calibrator every hour) gives an accuracy of $2^{\prime \prime}$ under most conditions.

\footnotetext{
${ }^{1}$ The National Radio Astronomy Observatory is operated by Associated Universities, Inc., under cooperative agreement with the National Science Foundation.
} 
With a system temperature of $100 \mathrm{~K}$ and an efficiency of $15 \%$, the VLA at $7 \mathrm{~mm}$ - with 10 antennas, $2-50 \mathrm{MHz}$ bandwidths, 10 minutes integration-will have an rms sensitivity of $1.9 \mathrm{mJy}$. This sensitivity should cover the frequency range 40.0 to $49.0 \mathrm{GHz}$. Observations outside of this range will have diminished sensitivity, but the drop-off is unknown until the receiver system is complete. The primary beam size of the telescopes is about $75^{\prime \prime}$, and in the A-configuration the resolution will be $.05^{\prime \prime}$. For radio emission with structure greater than $1^{\prime \prime}$ more compact configurations must be used to image the source.

The two major lines within the $7 \mathrm{~mm}$ range of the VLA are the SiO maser lines at $42.519,42.820,43.122,43.423 \mathrm{GHz}$ and the CS line at $48.991 \mathrm{GHz}$. The isotopic $\mathrm{C}^{34} \mathrm{~S}$ at 48.206 and $\mathrm{C}^{33} \mathrm{~S}$ at $48.585 \mathrm{GHz}$ are also available. Other lines are $\mathrm{HNCO}$ at $43.963, \mathrm{HCCCN}$ at $45.490, \mathrm{CH}_{3} \mathrm{OH}$ at $48.375 \mathrm{GHz}$. Studies of $\mathrm{SiO}$ masers are important in determining the kinematics of evolving stars, whereas the CS emission occurs in dense molecular clouds $\left(>10^{4} \mathrm{~cm}^{-3}\right)$.

Continuum observations will be useful for sources which have high-frequency turnovers and significant dust emission. Because of the reduced sensitivity at $7 \mathrm{~mm}$ compared with $1.3 \mathrm{~cm}$, continuum observations are not expected to be a major part of the observing. However, when used as a phased-element in the VLBA (see next section), the VLA will afford much greater sensitivity. Proposals for use of the VLA in the $7 \mathrm{~mm}$ band are now being accepted at NRAO.

\section{THE VLBA AT MILLIMETER WAVELENGTHS}

The VLBA, an array of ten $25-\mathrm{m}$ antennas which spans over $8500 \mathrm{~km}$, is nearing completion. The array lies mostly in an e/w direction, from Saint Croix in the Virgin Islands to Mauna Kea in Hawaii. Most of the antennas are in the western part of the USA. The array has been designed to operate in nine observing bands, from $90 \mathrm{~cm}$ to $7 \mathrm{~mm}$, and any frequency change can be made in about 20 seconds. There is also a dual $11 \mathrm{~cm} / 3.6 \mathrm{~cm}$ capability. The control center of the array is in Socorro, New Mexico and the remote sites are staffed by two employees who change video recording tapes and repair the electronic modules. The data on the video tapes are transported to Socorro for correlation and processing.

The original design of the VLBA does include the $7 \mathrm{~mm}$ band. Because of the high resolution of the VLBA, the SiO maser line will be the most important species to be studied; hence the nominal frequency range of the VLBA between 42.3 and $43.5 \mathrm{GHz}$. Reduced sensitivity will be available outside of this range. The antenna efficiency is about $25 \%$, the system temperature is $100 \mathrm{~K}$, and the pointing accuracy is about $5^{\prime \prime}$. Over a typical coherence time of 30 seconds with a one $\mathrm{MHz}$ bandwidth, the rms noise on a baseline is about $0.4 \mathrm{Jy}$. Sources at least this strong can be self-calibrated in order to form images. The resolution of the full VLBA is $0.0002^{\prime \prime}$.

There are post-construction plans to implement the VLBA at $86 \mathrm{GHz}$. This upgrade will depend on the availability for funds over the next five years for the multitude of VLBA enhancements. However, the VLBA has been designed with $86 \mathrm{GHz}$ in mind; space is available in the feed ring and in the electronic racks, and the pointing accuracy of the antennas should be satisfactory. (On-line methods for correcting the pointing of the Green Bank Telescope, now under construction, may also be implement on the VLBA.) At these high frequencies 
and high resolutions, special calibration techniques for imaging will have to be developed.

Superb images of $\mathrm{SiO}$ masers around stars have already been made with the VLBA. Comparison of the transverse kinematics measured with the VLBA and the Doppler motion yield a statistical parallax of the star. With the high resolution images of the continuum radiation, the VLBA will probe into the heart of powerful galaxies and quasars. Based on previous observations at $7 \mathrm{~mm}$ with global VLBI arrays, the inner hundred parsec regions of many extragalactic sources change on time scales of months with ejecta appearing to travel more than the speed of light. Only with a stable, high-resolution array which is devoted to full-time high resolution observations, can sufficiently detailed and frequent observations of these objects be made.

A major goal of the VLBA is to make VLBI observations accessible to all astronomers regardless of their previous observational experience. In the past $\mathrm{mm}$-wave VLBI has been extremely arduous, from the scheduling of the observations, the coordination of the various observatories, the correlation of the data, and the production of the images. The VLBA, after the normal debugging and improvements in the first years, should be nearly as easy to use as the VLA. 